\title{
EL RETO DE LA EQUIDAD URBANA-RURAL EN EL ACCESO AL AGUA DE USO DOMÉSTICO EN COLOMBIA
}

\author{
The Challenge of Urban-Rural Equity in \\ Water Access for Domestic Use in Colombia
}

\begin{abstract}
MARÍA CECILIA ROA-GARCÍA ${ }^{1,2}$, ANDREINA PULIDO-ROZO
1University of British Columbia, Faculty of Land and Food. MCML 156, 2357 Main Mall Vancouver, Canada. ${ }^{2}$ FundaciónEvaristo García. Parcelación Colinas de Arroyohondo, Casa 11, Km 1 vía a Dapa. Cali-Colombia. ${ }^{3}$ Trent University, Sustainability Studies M.A. 1600 W Bank Dr, Peterborough, ON K9J7B8 Ontario, Canada.
\end{abstract}

E-mail: croa09@gmail.com

andreinapulido@trentu.ca

Recibido: 29 de Enero de 2014

Aceptado: 22 de Febrero de 2014

\begin{abstract}
Resumen
El mecanismo de subsidios para el acceso al agua en Colombia ha tenido una aplicación diferente en zonas urbanas y rurales. Mientras que en las zonas urbanas los subsidios son administrados por grandes empresas comerciales, en las zonas rurales los intermediarios son organizaciones comunitarias que en la mayoría de los casos no tienen capacidad de gestión para tramitar los subsidios ante las entidades del Estado. En este artículo presentamos cinco ejemplos de organizaciones comunitarias con diversos niveles de gestión y analizamos las dificultades que han enfrentado para acceder a los subsidios a los que tienen derecho sus suscriptores. Argumentamos que aunque algunas organizaciones ven una ventaja en mantener la independencia del Estado y optan por no acceder a los subsidios, el acceso a estos recursos por parte de las organizaciones comunitarias del agua es necesario no solo para garantizar su sostenibilidad, sino para contribuir a reducir la brecha de inequidad entre zonas urbanas y rurales. Las organizaciones comunitarias pueden ser una solución financieramente más eficiente que las grandes empresas público/privadas y sus economías de escala para suplir agua potable en zona rural a bajo costo.
\end{abstract}

Palabras claves: Subsidios, Agua rural, Equidad, Sostenibilidad.

\begin{abstract}
The subsidy mechanism for water access in Colombia has been applied differently in urban and rural areas. While in urban areas the subsidies are administered by large commercial enterprises, in rural areas the intermediaries between communities and the state are community organizations that in most cases have limited capacity to demand these resources from the state. In this article we present five examples of community organizations with diverse managerial capabilities and analyze the difficulties they have faced to access water subsidies their subscribers are entitled to. We argue that although some organizations see an advantage in maintaining the independence from the state and choose not to embark on the quest for subsidies, access to these resources by community organizations is necessary not only to ensure their sustainability but to help reduce the inequality gap between urban and rural areas. Community organizations may be a more financially efficient solution than large enterprises and their economies of scale to supply low cost drinking water in rural areas.
\end{abstract}

Keywords: Subsidies, Rural water, Equity, Sustainability. 


\section{Equidad Urbana-Rural en el Acceso al Agua}

\section{INTRODUCCIÓN}

Colombia ha hecho avances significativos en relación con otros países de América Latina, al crear un mecanismo de equidad, solidaridad y redistribución para el acceso al agua de consumo doméstico. La ley de servicios públicos de 1994 describe los subsidios como un mecanismo de equidad a través del cual las familias de bajos ingresos pueden acceder a los servicios públicos por valores subsidiados. Sin embargo 20 años después de aprobada la ley, los subsidios no llegan a las zonas rurales donde las organizaciones que prestan el servicio son comunitarias y sin ánimo de lucro, mientras que si son otorgados a las grandes empresas público/privadas que incluyen en sus cálculos de tarifas los márgenes de rentabilidad de capital. Este desbalance no solo ha contribuido a aumentar la brecha entre zonas rurales y urbanas, sino que también ha tergiversado una herramienta diseñada para la equidad. El propósito de este artículo es mostrar desde la perspectiva de las organizaciones comunitarias del agua, el dilema entre luchar por los subsidios haciendo valer las conquistas de la constitución de 1991 y haciendo uso de las oportunidades que ofrece la ley 142 de 1994, o permanecer al margen de ellos y mantener la independencia y el esfuerzo comunitario.

Para acceder a los subsidios las organizaciones prestadoras de servicios de agua deben cumplir con un número importante de requisitos, entre los cuales se destacan dos: la estratificación de los usuarios y el cobro de tarifas calculadas con la metodología de la Comisión Reguladora de Agua (CRA). La estratificación de los usuarios es una responsabilidad de los municipios, pero una gran proporción de las zonas rurales y peri-urbanas no han sido estratificadas, lo que se ha convertido en un serio obstáculo para el acceso a los subsidios. El cálculo y cobro de tarifas de acuerdo con el método de la CRA implican contabilizar todos los costos en que se incurren, con el fin de que las organizaciones puedan mantener sistemas de información que contribuyan con su sostenibilidad financiera y que se conviertan en la base para el cálculo de los subsidios y las contribuciones de los estratos.

Una vez las organizaciones conocen sus costos incurridos, han aprobado las tarifas de acuerdo con el método de la CRA y conocen los mecanismos para acceder a los subsidios para sus usuarios, tienen la opción de no emprender la lucha legal por este derecho. En este artículo mostramos a través de cinco ejemplos las posturas de las organizaciones frente a los subsidios, las dificultades para acceder a ellos, la negligencia de las administraciones municipales en dar cumplimiento a la ley y la lucha legal que se debe dar para acceder a este derecho. Argumentamos que el acceso a los subsidios puede llegar a ser un importante soporte para el modelo comunitario de agua. Los subsidios pueden contribuir a remunerar el trabajo que por décadas han aportado voluntariamente administradores de estos sistemas, contribuir a cerrar la brecha de inequidad entre lo urbano y lo rural y pueden estimular la capacitación de miembros activos de la comunidad en el manejo del agua y en el uso de la ley para defender un modelo de gestión que acerca el agua a la sociedad.

Con este análisis presentamos algunos insumos para responder preguntas tales como: ¿cómo puede el Estado mejorar el mecanismo de trámite y desembolso de los subsidios para que el acceso a los servicios públicos sea una herramienta efectiva para una sociedad más equitativa? ¿Pueden los sistemas comunitarios de abastecimiento de agua acceder a estos recursos sin perder su carácter comunitario? Y ¿cuáles son los riesgos para los sistemas comunitarios al recibir subsidios del Estado?

\section{MÉTODOS}

Se presenta una síntesis de la ley 142 de 1994 con relación a la equidad y la sostenibilidad económica de las organizaciones comunitarias de agua y un análisis de su cumplimiento. Se presentan también las reglas establecidas por la Comisión Reguladora de Agua (CRA) a través de la resolución 287 de 2004 que establece la forma del cálculo de tarifas tanto para empresas como para organizaciones comunitarias; y las exigencias de la ley 142 con relación al trámite y desembolso de los subsidios. También se presentan los mecanismos legales disponibles para las organizaciones comunitarias a fin de exigir el cumplimiento del pago de subsidios por parte de los municipios.

Los datos que soportan el análisis del cumplimiento de la ley provienen de dos fuentes: una encuesta realizada a 46 organizaciones comunitarias prestadoras de servicios de agua en los departamentos de Cauca, Risaralda y Valle del Cauca; y el Sistema Único de Información (SUI) cuya información está disponible al público en la página web de 
la Superintendencia de Servicios Públicos Domiciliarios (SSPD). Del SUI se bajaron las listas de las organizaciones con más de 2500 suscriptores con la información correspondiente a subsidios y contribuciones para cruzarla con la información correspondiente a lo recibido por parte del Fondo de Solidaridad y Redistribución de Ingresos (FSRI).

El análisis de las posibles causas por las que los mecanismos de equidad y sostenibilidad propuestos en la ley son poco aplicables, se presenta con base en un análisis de los estados financieros y la información detallada de cinco organizaciones comunitarias del Valle del Cauca (Acuasur, Acuabuitrera, Golondrinas y La Sirena) y Risaralda (Tribunas Córcega). Con base en esta información se presenta una comparación entre las tarifas actuales y las tarifas calculadas con el método de la CRA, y con las tarifas de una empresa urbana de gran tamaño. Tomamos las tarifas de las Empresas Municipales de Cali - EMCALI disponibles en su página web.

\section{La equidad y la sostenibilidad de las organizaciones comunitarias en la ley de servicios públicos y sus mecanismos}

La ley 142 de 1994 estableció los siguientes principios para el manejo de los servicios públicos: neutralidad, solidaridad, redistribución, transparencia, eficiencia económica y suficiencia financiera. Los principios de solidaridad y redistribución buscan crear las condiciones para que los más pobres puedan acceder a los servicios públicos con tarifas por debajo de los costos y que los más ricos de la sociedad contribuyan a cubrir estos subsidios. Los mecanismos creados para operacionalizar estos principios son dos: 1) las tarifas diferenciadas de acuerdo con la estratificación socioeconómica para que los miembros de la sociedad con mayor capacidad de pago (estratos 5 y 6, industriales y comerciales) contribuyan a subsidiar el servicio de los miembros más pobres (estratos 1, 2 y 3); y 2) el FSRI al que contribuyen los estratos altos y el gobierno central para cubrir el déficit entre subsidios y contribuciones. En general los usuarios que contribuyen al FSRI están concentrados en zonas urbanas, mientras que las organizaciones que prestan servicios en zonas rurales y peri-urbanas que son entre 12000 (Fernández 2004) y 25000 (Pérez 2001) organizaciones, atienden mayoritariamente a familias de estratos socioeconómicos bajos. Ni siquiera las empresas prestadoras del servicio en las grandes ciudades logran un balance entre subsidios y contribuciones.

Por este desbalance, el Estado es el mayor contribuyente al FSRI al que los acueductos aplican anualmente para poder cubrir sus operaciones y mantener la infraestructura. A pesar de que el objetivo del FSRI es la equidad en el acceso al agua, el acceso al fondo no es equitativo. Una proporción mínima de los acueductos rurales y peri-urbanos acceden a estos recursos. De acuerdo con la información disponible en el portal del SUI de la SSPD, el $67 \%$ de las empresas de más de 2500 conexiones que solicitaron los subsidios recibieron transferencias del FSRI en 2010 y 58\% en 2011, en contraste con sólo el $11 \%$ por parte de las organizaciones comunitarias encuestadas. Esto, enmarcado en un escenario nacional inequitativo donde la cobertura de agua potable en zonas urbanas alcanza el $96 \%$ mientras que en zonas rurales es sólo del 57\% (Departamento Administrativo Nacional de Estadística -DANE 2010), amplía más la brecha entre zonas rurales y urbanas. No es posible usar el SUI para determinar el porcentaje de organizaciones de menos de 2500 conexiones que reciben subsidio, pues se estima que menos del $15 \%$ de las organizaciones pequeñas reportan información al SUI. Pero considerando que reportar información al SUI es un requisito para acceder a los subsidios se puede deducir que ninguna de las organizaciones que no reportan, están recibiendo los subsidios. En estas cifras se manifiestan las dificultades que enfrentan las organizaciones comunitarias para ser las intermediarias entre las familias de estratos bajos y el FSRI, cuyo fin ulterior es contribuir a un acceso equitativo al agua.

Otro factor de inequidad en la práctica versus la norma, es el costo excesivo del agua potable respecto al ingreso de las familias en zona rural. Teniendo en cuenta el límite normativo del $3 \%$ de los ingresos familiares en servicio de agua, para no comprometer el gasto en otros bienes $y$ servicios, aún con subsidio el gasto en agua de los hogares colombianos es excesivo para aquellos que pertenecen a los dos quintiles más bajos de la distribución (Meléndez 2008).

Por otro lado la ley 142 en su artículo 99.8 establece que la transferencia de los fondos de debe hacer en un plazo de 30 días contados desde la fecha de la factura a cargo del municipio. Para asegurar la transferencia, las organizaciones deben firmar contratos con el municipio. En caso de que las organizaciones cumplan con todos los requisitos y el 
municipio sea negligente en la aplicación de las normas relacionadas con los subsidios, pueden acudir al artículo 99.4 de la ley 142 de 1994 el cual establece que el presidente y los gobernadores podrán suspender a los alcaldes si estos no están cumpliendo con cualquiera de las normas relacionadas con ellos.

En las secciones siguientes analizamos de manera específica dos de los principales obstáculos para el acceso a los subsidios: la estratificación rural y el cálculo y cobro de tarifas con la metodología de la CRA, a partir de algunos ejemplos de organizaciones comunitarias que han participado en procesos de estratificación de sus comunidades y/o que han realizado un estudio de costos y tarifas.

\section{Estratificación para la redistribución}

En muchos casos las organizaciones clasifican sus usuarios de acuerdo con su capacidad de pago y no necesariamente de acuerdo con el sistema de estratificación exigido por el gobierno nacional. El sistema de estratificación colombiano, cuyo principal objetivo ha sido la equidad en el acceso a servicios públicos entre grupos sociales con diferente capacidad de pago, ha sido cuestionado ampliamente especialmente porque no captura las diferencias en capacidad de pago, porque ha contribuido a mayor segregación social (Martínez-Alonso 2004, Mina-Rosero 2004, Meléndez 2008) y también porque la estratificación se convirtió en la expresión de las voluntades e intereses económicos y políticos frente a lo público como se evidencia particularmente en la estratificación rural (Álzate 2006). Aquí no nos ocuparemos de cuestionar el sistema de estratificación, sino de hacer algunas observaciones sobre las dificultades que enfrentan las organizaciones comunitarias particularmente rurales y peri urbanas cuando solicitan la estratificación a sus respectivos municipios como requisito para acceder al FSRI.

En la ciudad de Cali, las zonas peri urbanas donde se ubican los acueductos de La Sirena y Acuabuitrera no han sido estratificadas de acuerdo con el método propuesto por el DANE. De acuerdo con este método para las zonas rurales, al igual que para zonas urbanas se debe contar con la base predial catastral. Esta base se usa para que el departamento de planeación municipal compare el predio con la zona geoeconómica donde se encuentra, y en algunos casos con la información que provean los prestadores de servicios públicos sobre sus clientes o asociados. En las zonas rurales de Cali estos procesos no se han adelantado. Aun si las organizaciones comunitarias tuvieran información completa de sus comunidades (por ejemplo el número predial) que en algunos casos es difícil de obtener por la renuencia de la gente a dar información sobre sus predios por temor a que suban sus tarifas o sus impuestos, la falta de la base predial catastral se ha convertido en un gran obstáculo para este proceso. Para el caso de la zona rural de Pereira donde se encuentra Tribunas Córcega, la presión ejercida por las organizaciones comunitarias de la zona rural, motivó a la alcaldía a emprender la estratificación rural que se hizo con la participación de las organizaciones comunitarias.

En algunos casos donde la estratificación no se ha realizado formalmente las organizaciones comunitarias han optado por hacer estratificaciones con base en otros prestadores de servicios públicos (e.g. Energía) u otros métodos de estratificación sugeridos por los gobiernos municipales o creados por las mismas organizaciones. Existen organizaciones comunitarias que tienen acceso a subsidios en zonas rurales que aún no están debidamente estratificadas según las exigencias del DANE.

\section{TARIFAS Y SOSTENIBILIDAD}

Una de las principales deficiencias de los sistemas comunitarios de abastecimiento de agua tiene que ver con temas administrativos relacionados con la recuperación de los costos (Tamayo \& García 2006). Una gran mayoría de las organizaciones comunitarias cobran tarifas con base en procesos de negociación con las comunidades y no con base en los costos reales de operación, mantenimiento y administración, y mucho menos con base en las necesidades de reposición y actualización de infraestructura.

En la Figura 1 se observa la distribución de la población atendida por las cinco organizaciones estudiadas. De las cinco, solamente Tribunas Córcega tiene todos sus suscriptores formalmente estratificados, y aunque la población no está concentrada en los estratos más bajos, los contribuyentes son solo el $10 \%$ del total de suscriptores. Para Golondrinas, La Sirena y Acuasur, entre el 95\% y el $99 \%$ de sus suscriptores son de las categorías 1 y 2, mientras que para Acuabuitrera el 39\% están en las categorías 1 a 3 y 
el 51\% en la categoría 4. Esta categorización indica que estas organizaciones están prestando servicios a una población de bajos ingresos que tiene derecho a recibir subsidios a través del FSRI.

En esta sección mostraremos las diferencias entre las tarifas que cobran las organizaciones estudiadas y las que resultarían al usar el método de la CRA y analizamos las dificultades para hacer la transición hacia estas tarifas.

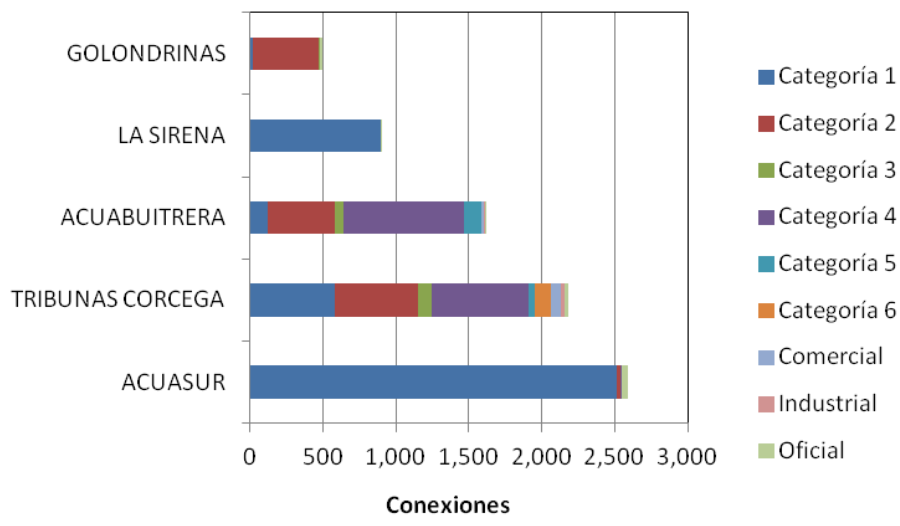

Figura 1. Tamaño y distribución de los suscriptores de las organizaciones (Categoria 1 corresponde a los usuarios de bajos ingresos y la categoria 6 a los usuarios de altos ingresos).

Con base en el principio de suficiencia financiera, la CRA reglamentó las tarifas para todas las organizaciones prestadoras de servicios de acueducto y alcantarillado. El principio busca que las organizaciones sean sostenibles en términos financieros, recaudando tarifas tanto para la administración de las operaciones, como para el mantenimiento y las expansiones de infraestructura. La resolución 287 de 2004 de la CRA presenta las fórmulas simplificadas para el cálculo de las tarifas de las organizaciones de menos de 2500 conexiones en dos componentes: un cargo fijo y un cargo por consumo. El cargo fijo se calcula como el Costo Medio Administrativo (CMA) de la siguiente manera:

$$
\mathrm{CMA}=\frac{\text { Sumatoria de Gastos Administrativos Anuales }}{\text { Número de Suscriptores Facturados }}
$$

El cargo por consumo se calcula con la siguiente fórmula: Cargo por Consumo $=$ Costo Medio de Operación + Costo Medio de Inversión + Costo Medio por Tasa Ambiental

$$
\mathrm{CC}=\mathrm{CMO}+\mathrm{CMI}+\mathrm{CMT}
$$

Donde,

$$
\text { CMO }=\frac{\text { Sumatoria de Costos de Operación Anuales }}{\mathrm{m} 3 \text { producidos al año }(1-\mathrm{P} *)}
$$

$$
\begin{gathered}
\text { CMI }=\frac{\text { Sumatoria de Proyección de inversión a Corto Plazo }}{\mathrm{m} 3 \text { producidos al año }(1-\mathrm{P} *)} \\
\text { CMT }=\frac{\text { Tasa por uso del agua por cada } \mathrm{m} 3}{1-\mathrm{P} *}
\end{gathered}
$$

La tasa por uso del agua corresponde al aporte por la concesión de agua otorgada por la Corporación Autónoma Regional (autoridad ambiental regional).

$\mathrm{P}^{*}=$ Nivel máximo aceptable de pérdidas de agua definido por la CRA

$\mathrm{P}^{*}=30 \%$

Con estas fórmulas y con base en los estados financieros de las organizaciones estudiadas, se calcularon las tarifas. La Figura 2 resume las diferencias en el cargo fijo (actuales y con método CRA) para los estratos 1 a 4 y la Figura 3 las diferencias en el cargo por consumo para los mismos estratos.

Asumiendo que las categorías de las organizaciones corresponden a los estratos, se observa que las organizaciones comunitarias que aún no cobran tarifas CRA tienen en la mayoría de los casos cargos fijos por encima de los que resultan de los cálculos con el método CRA. La excepción es Tribunas Córcega que ya cobran tarifas con el método CRA, por lo cual no existen diferencias.

En la Figura 3 se observan interesantes diferencias entre las organizaciones. Golondrinas mantiene un cargo por consumo que es casi la mitad del que cobraría con el método CRA en el estrato 2 donde se agrupa el 91\% de sus usuarios. 
Acuabuitrera no cobra cargos por consumo por debajo de los primeros $20 \mathrm{~m}^{3}$, mientras que La Sirena y Acuasur tienen cargos por consumo superiores a los del método CRA. Para estas dos organizaciones las diferencias son mayores especialmente en el estrato 1 donde los cargos actuales son más del doble de los cargos con el método CRA.

En la Figura 4 se muestra lo que pagaría un usuario en cada estrato si tuviera un consumo mensual de $20 \mathrm{~m} 3$. Esta comparación permite analizar el manejo que se le ha dado a las tarifas dentro de la organización comunitaria y es un indicador de la sostenibilidad financiera de la organización. Por ejemplo contrasta que Golondrinas esté cobrando tarifas inferiores a las tarifas CRA en el estrato 2 (donde se concentra el $91 \%$ de sus suscriptores) mientras que en el estrato 1 cobra una tarifa superior a lo que cobraría con el método CRA. La Sirena y Acuasur cobran tarifas superiores a las que cobrarían con el método CRA. Lo que refleja esta comparación es que organizaciones como Golondrinas cubren costos básicos de operación y administración, pero no cubren costos relacionados con inversiones y se mantienen en una precaria situación financiera que pone en riesgo su sostenibilidad. La Sirena y Acuasur, por otro lado, tienen tarifas actuales superiores a las tarifas CRA, pues no han podido acceder a los subsidios y sus usuarios están pagando tarifas por encima de lo que deberían pagar de acuerdo con el método CRA. Para Acuabuitrera, que no
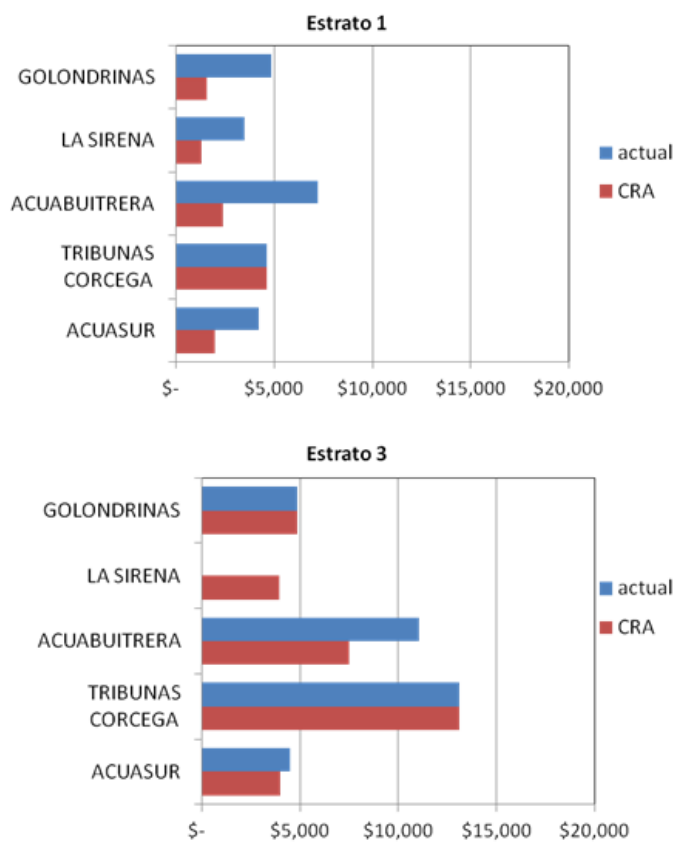

Figura 2. Comparación entre cargos fijos actuales y los calculados con las fórmulas CRA (pesos por mes). cobra cargo por consumo por debajo de $20 \mathrm{~m}^{3}$, esta figura muestra el impacto del volumen de agua consumido en los costos, y que el cargo fijo no alcanza a cubrir estos costos, a pesar de ser significativamente más altos de lo que serían por el método CRA para todos los estratos (Figura 2).

El tipo de obstáculo para hacer la transición hacia tarifas CRA entonces depende de la cobertura actual de los costos. Para una organización como Golondrinas, la transición implica una socialización con la comunidad puesto que el cargo por el metro cúbico de consumo se incrementaría en un $88 \%$ para el estrato 2 y en un $32 \%$ considerando los dos cargos para un consumo de $20 \mathrm{~m}^{3}$ al mes. Lo mismo pasaría en Acuabuitrera que debería iniciar los cobros por consumo y reducir los cargos fijos. Sin embargo la estratificación podría cambiar la composición actual y ubicar un porcentaje mayor de usuarios en el estrato 1 donde las tarifas serían menores por un menor cargo por consumo. Para organizaciones como Acuasur y La Sirena tanto el cargo fijo como el cargo por consumo en los estratos 1 a 4 ( 1 y 2 para Acuasur que tiene el $98 \%$ de sus suscriptores en estos estratos) bajarían significativamente con un impacto muy positivo en el estrato 1. En las Tablas 1, 2, 3 y 4 se presenta la comparación entre las tarifas actuales y las calculadas con el método CRA para Acuabuitrera, Golondrinas, Acuasur y La Sirena respectivamente.
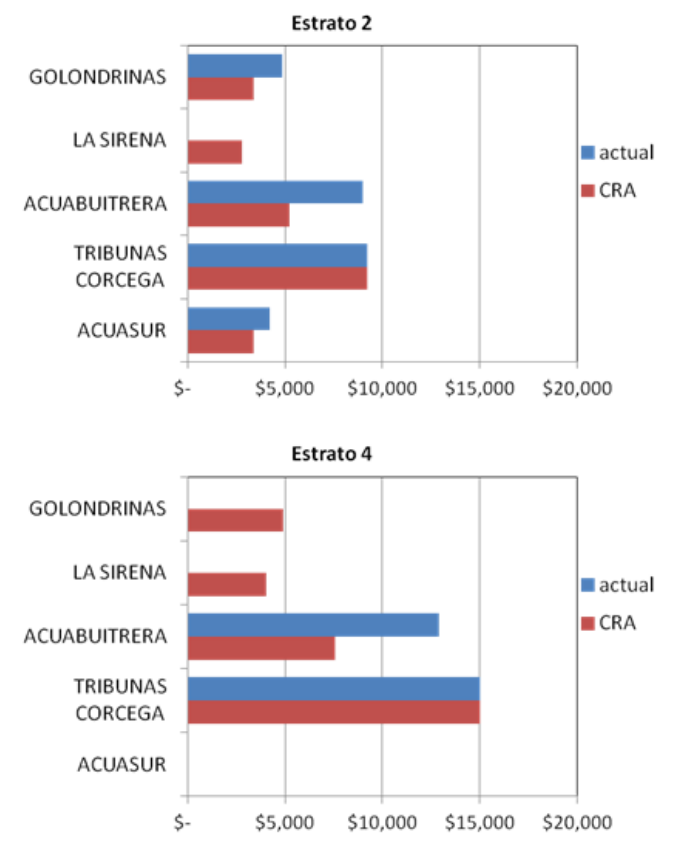
Ambiente y Sostenibilidad 2014 (4): 3-15

Revista del Doctorado Interinstitucional en Ciencias Ambientales

ISSN: 2339-3122

Acuabuitrera y Golondrinas reconocen que el cambio de las tarifas es necesario para cubrir los costos de operación del acueducto y asegurar su sostenibilidad, pero advierten que el mayor reto consiste en socializarlo con la comunidad. El incremento de tarifas ya ocurrió en acueductos comunitarios como Tribunas Córcega, pero el proceso de incremento de tarifas tuvo que ser gradual y tomó varios años. "En

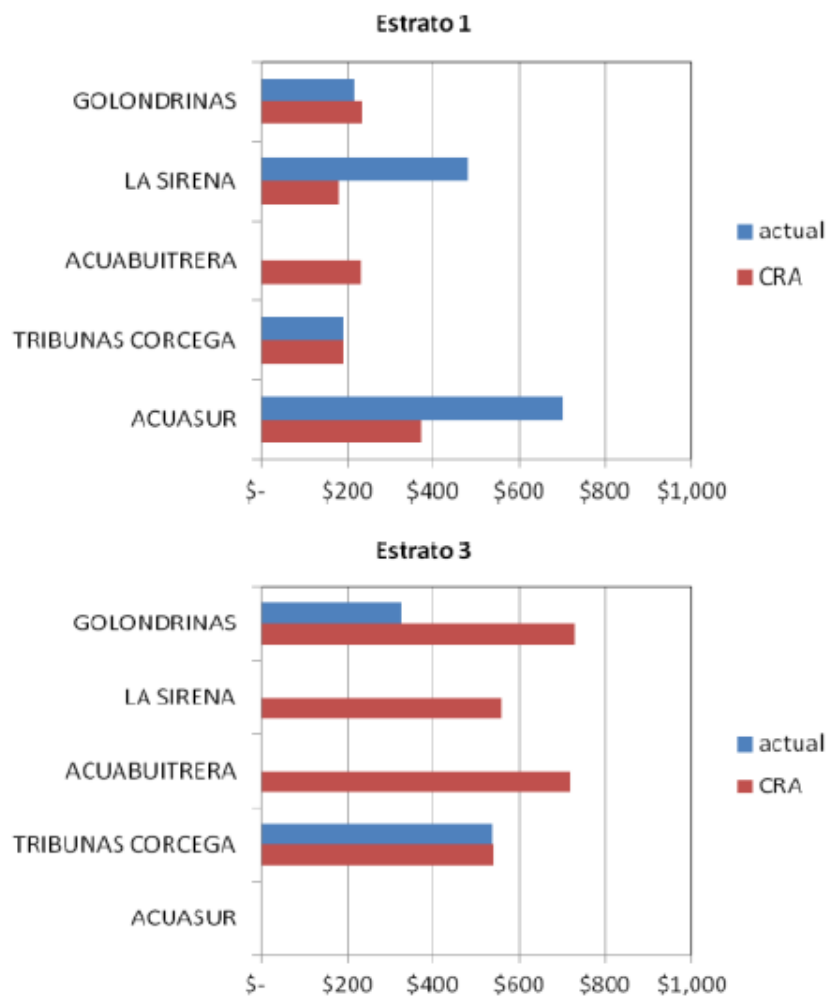

Tribunas desde que llegó el Dr. Oscar Gómez a la gerencia (2003) se recalculó la tarifa y había un rezago grande, por tal motivo se hicieron incrementos mensuales de 1\% hasta 2010 y a partir de ese año se hicieron incrementos de $6 \%$ decidido por Asamblea de Delegados" - Adriana Vélez, Tesorera, Tribunas Córcega, 2013.

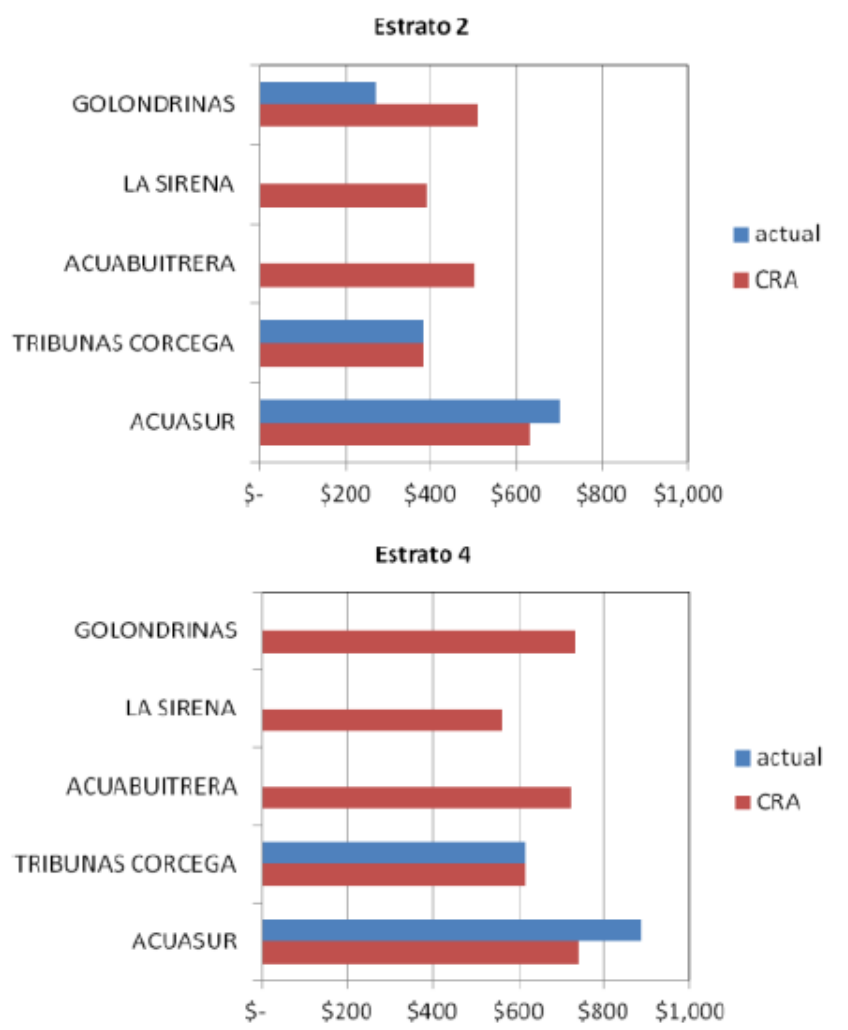

Figura 3. Comparación en cargos por consumo actuales y los calculados con las fórmulas CRA (pesos por mes por 20 m³)

Una vez se han implementado las nuevas tarifas, Tribunas Córcega recalcula las tarifas año a año así: "El cálculo de la tarifa con base en los costos operacionales y administrativos se hace cada 5 años según la metodología de la CRA, los cambios en la tarifa después del cálculo son muy grandes, por lo tanto se prorratea o acomoda para hacer incrementos paulatinos año a año de manera que no afecte tanto al usuario. El incremento anual es decidido por Asamblea de Delegados" - Adriana Vélez, Tesorera, Tribunas Córcega, 2013.

De acuerdo con esta experiencia, el incremento de tarifas debe ser fruto de un proceso de socialización y participación con la comunidad y debe hacerse de manera gradual.
Tabla 1. Acuabuitrera: Comparación de tarifas actuales y método CRA

\begin{tabular}{lrrr}
\hline Estrato & $\begin{array}{c}\text { No. } \\
\text { subscriptores }\end{array}$ & $\begin{array}{c}\text { Tarifa actual } \\
\text { comparable } \\
\text { por 20 } \mathrm{m}^{3} \\
\text { (pesos) }\end{array}$ & $\begin{array}{c}\text { Tarifa CRA } \\
\text { comparable por 20 } \\
\mathrm{m}^{3} \text { (pesos) }\end{array}$ \\
\hline Estrato 1 & $8 \%$ & 7,200 & 12,198 \\
Estrato 2 & $28 \%$ & 9,000 & 15,222 \\
Estrato 3 & $3 \%$ & 11,050 & 21,841 \\
Estrato 4 & $51 \%$ & 12,900 & 22,061 \\
Estrato 5 & $7 \%$ & 22,900 & 33,092 \\
Estrato 6 & $0.5 \%$ & 0 & 35,298 \\
Comercial & $1 \%$ & 31,150 & 33,092 \\
Industrial & $1 \%$ & 24,675 & 28,680 \\
Oficial y & & & 22,061 \\
Especial & $0.5 \%$ & 12,900 & 9
\end{tabular}


Organizaciones como La Sirena y Acuasur que cobran tarifas más altas que las que resultarían del método CRA para más del $97 \%$ de sus suscriptores, expresan su preocupación al tener que bajar sus tarifas antes de recibir los subsidios. Las demoras en el pago de los subsidios los harían extremadamente vulnerables y fácilmente afectarían la operación del servicio de agua a sus comunidades, incluso hasta el punto de llevarlos a la quiebra. Obtener los subsidios haría muy dependientes a organizaciones comunitarias como Acuasur y La Sirena, perdiendo así control sobre la disponibilidad de sus ingresos.

De acuerdo con las instrucciones del decreto 1013 del 2005 las organizaciones deben pasar a la respectiva alcaldía a través del FSRI, antes del 15 de Julio de cada año una estimación para el año siguiente de los recaudos por aportes solidarios y de los subsidios a otorgar. A pesar de que la ley de servicios públicos establece en su artículo 99.8 que la transferencia de fondos desde el FSRI hacia las organizaciones debe hacerse en un plazo máximo de 30 días
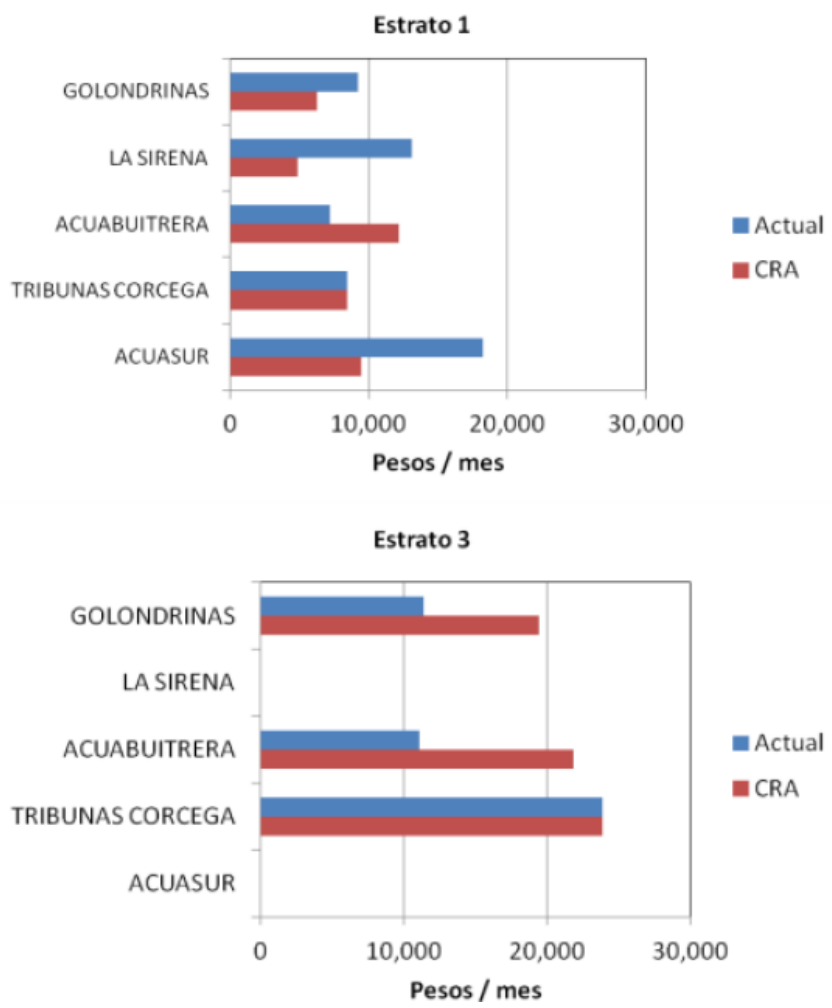

desde la fecha de la factura a cargo del municipio, en la experiencia de las organizaciones los montos provenientes del FSRI por lo general son desembolsados a mediados del año siguiente, es decir que durante la primera mitad del año las organizaciones deben financiar sus operaciones con recursos de terceros, encareciendo el servicio al crear dependencia del sector financiero. El obstáculo para pasar a cobrar tarifas CRA para organizaciones como Acuasur radica en que los ingresos se verían reducidos antes de recibir los subsidios, afectando el flujo de caja de la organización y obligándola a financiarse a través de créditos bancarios. Para Tribunas Córcega esta situación es manejable debido a que su flujo de caja es mayor por el mejor balance entre subsidios y contribuciones (importante número de usuarios de estrato 6 , comerciales e industriales), pero su gerente considera que depender de las entidades financieras para financiar las operaciones de los acueductos mientras el Estado desembolsa los subsidios hace que el sistema sea inviable para las organizaciones comunitarias.

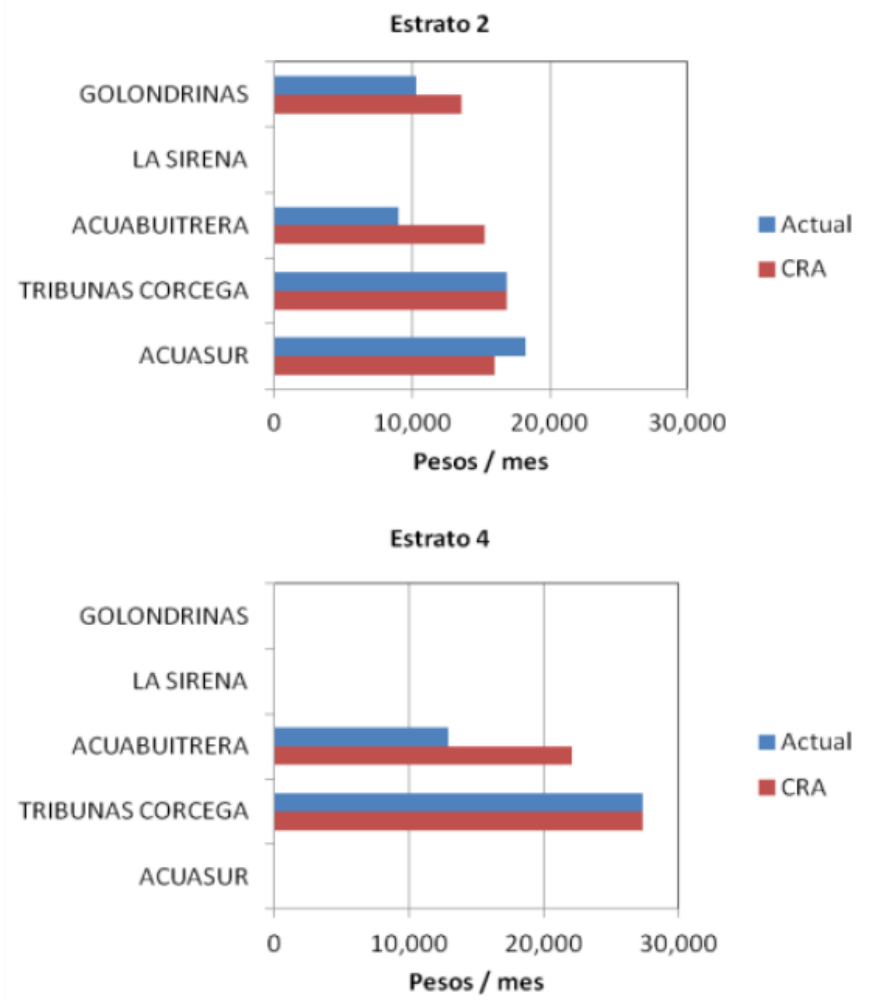

Figura 4. Comparación entre tarifa actual y tarifa CRA por estrato para $20 \mathrm{~m}^{3}$ mensuales (pesos por mes).

La falta de acceso a los subsidios se convierte entonces en un círculo vicioso, pues al no tener acceso a ellos, las organizaciones deben mantener costos bajos, dejan de incluir inversiones en sus costos y en muchos casos 
mantienen bajos costos de administración a través del trabajo voluntario de miembros de la comunidad. Para organizaciones como La Sirena, ésta es la fortaleza de las organizaciones comunitarias: su independencia de los recursos del Estado para el manejo del agua. Como puede verse en las figuras 1, 2, 3 y 5 La Sirena es una organización diferente a las demás, al tener uno de los cargos fijos más bajos de la muestra y que al cobrar tarifas CRA tendría las tarifas más bajas en todos los estratos. Esta situación se debe a la eficiente tecnología empleada (planta de filtración en múltiples etapas que es poco intensiva en mano de obra y en químicos para tratar el agua) y principalmente a que un gran porcentaje de los costos administrativos son asumidos por la comunidad. Un estimado realizado con los administradores del acueducto arroja que si la gerencia y algunas funciones administrativas fueran remuneradas al igual que la asesoría en temas técnicos, los costos y gastos se incrementarían en un $46 \%$ lo cual se vería reflejado directamente en las tarifas. Esta misma situación se presenta en Golondrinas donde los costos no incurridos por la organización corresponden a un 46\%. En Acuabuitrera y Acuasur los porcentajes de costos no incurridos y no cubiertos por las tarifas son menores, pero de todas maneras significativos tal como se muestra en la tabla 5.

Tabla 2. Golondrinas: Comparación de tarifas actuales y con método CRA.

\begin{tabular}{lrrr}
\hline Estrato & $\begin{array}{c}\text { No. } \\
\text { subscriptores }\end{array}$ & $\begin{array}{c}\text { Tarifa actual } \\
\text { comparable } \\
\text { por 20 } \mathrm{m}^{3} \\
\text { (pesos) }\end{array}$ & $\begin{array}{c}\text { Tarifa CRA } \\
\text { comparable por 20 } \\
\mathrm{m}^{3} \text { (pesos) }\end{array}$ \\
\hline Estrato 1 & $4 \%$ & 9,180 & 6,279 \\
Estrato 2 & $91 \%$ & 10,260 & 13,540 \\
Estrato 3 & $0 \%$ & 11,340 & 19,427 \\
Estrato 4 & & & \\
Estrato 5 & & & \\
Estrato 6 & & & \\
Comercial & & & \\
Industrial & & & \\
Oficial y & $5 \%$ & 12,420 & \\
Especial & & & \\
\hline
\end{tabular}

En esta sección hemos mostrado la gran diferencia que haría en las tarifas de los estratos bajos el acceso a los subsidios por parte de organizaciones que han trasladado los costos a sus comunidades; y cómo en el mediano y largo plazo la sostenibilidad de las organizaciones comunitarias va a depender en gran medida del acceso que puedan tener a los subsidios que les permitan cubrir los costos que hasta ahora son subsidiados por voluntarios de las administraciones de estos sistemas.

Tabla 3. Acuasur: Comparación de tarifas actuales y con método CRA.

\begin{tabular}{lrrr}
\hline Estrato & $\begin{array}{c}\text { No. } \\
\text { subscriptores }\end{array}$ & $\begin{array}{c}\text { Tarifa actual } \\
\text { comparable } \\
\text { por 20 } \mathrm{m}^{3} \\
\text { (pesos) }\end{array}$ & $\begin{array}{c}\text { Tarifa CRA } \\
\text { comparable por 20 } \\
\mathrm{m}^{3} \text { (pesos) }\end{array}$ \\
\hline Estrato 1 & $97 \%$ & 18,200 & 9,414 \\
Estrato 2 & $1 \%$ & 18,200 & 16,003 \\
Estrato 3 & $1 \%$ & 22,217 & 18,827 \\
Estrato 4 & & & \\
Estrato 5 & & & \\
Estrato 6 & & & \\
Comercial & & & \\
Industrial & $1 \%$ & 22,217 & \\
Oficial y & & & \\
Especial & & & \\
\hline
\end{tabular}

Tabla 4. La Sirena: Comparación de tarifas actuales y con método CRA

\begin{tabular}{lccc}
\hline Estrato & $\begin{array}{c}\text { No. } \\
\text { subscriptores }\end{array}$ & $\begin{array}{c}\text { Tarifa actual } \\
\text { comparable } \\
\text { por 20 } \mathrm{m}^{3} \\
\text { (pesos) }\end{array}$ & $\begin{array}{c}\text { Tarifa CRA } \\
\text { comparable por 20 } \\
\mathrm{m}^{3} \text { (pesos) }\end{array}$ \\
\hline Estrato 1 & $99 \%$ & 13,100 & 4,880 \\
Estrato 2 & & & \\
Estrato 3 & & & \\
Estrato 4 & & & \\
Estrato 5 & & & \\
Estrato 6 & & & \\
Comercial & $0.5 \%$ & 13,100 & 19,824 \\
Industrial & & & \\
Oficial y & $0.5 \%$ & 13,100 & 15,249 \\
Especial & & & \\
\hline
\end{tabular}

\section{TARIFAS Y EQUIDAD}

La resolución 287 de 2004, siguiendo los lineamientos de la Ley 142, incorpora en las fórmulas de cálculo de las tarifas un componente que genera diferencias significativas entre las tarifas de las grandes empresas prestadoras de servicios en zonas urbanas y las de las organizaciones comunitarias. Se trata de los márgenes de rentabilidad financiera sobre las 
inversiones causadas (públicas o privadas). La Contraloría había propuesto un tope máximo de remuneración sobre el capital invertido del 12\%. Pero la resolución 312 de 2005 fijó esta remuneración de la siguiente manera: para las empresas de más de 25000 suscriptores, el margen de rentabilidad estará entre el $13.34 \%$ y el $13.92 \%$; y para las empresas que atiendan hasta 25000 suscriptores se ubicará entre $14.24 \%$ y
$14.58 \%$. Esta remuneración al capital invertido por parte de las empresas se ve reflejado en las tarifas de las grandes empresas, que son considerablemente más altas que las de las organizaciones comunitarias, como se puede apreciar en la Tabla 6 que resume los cargos fijos y cargos por consumo mensuales de las cinco organizaciones comunitarias en comparación con los de EMCALI.

Tabla 5. Costos y gastos no incurridos actualmente en relación con costos y gastos totales.

\begin{tabular}{|c|c|c|c|c|}
\hline $\begin{array}{l}\text { Costos y gastos no cubiertos } \\
\text { Millones de pesos (2012) }\end{array}$ & Golondrinas & La Sirena & Acuabuitrera & Acuasur \\
\hline Gerente o administrador & 16.8 & 22.0 & & \\
\hline Vicepresidente & 2.0 & & & \\
\hline Secretario & 0.1 & & & \\
\hline Tesorera / auxiliar contable & 4.9 & 10.8 & 8.8 & \\
\hline Representación de junta directiva & & & 7.6 & 2.5 \\
\hline Honorarios delegados asamblea & & & & 34.2 \\
\hline Muestras de calidad de agua & 9.0 & & 6.0 & \\
\hline Asesorías técnicas / jurídicas & & 13.8 & 5.9 & 36.5 \\
\hline Ingenieros de la comunidad & & 3.9 & & \\
\hline Capacitaciones por junta directiva & & & 4.0 & \\
\hline Mingas & & & 0.8 & 1.9 \\
\hline Total costos y gastos no cubiertos & 32.8 & 50.5 & 33.1 & 75.1 \\
\hline Total costos y gastos & 71.7 & 110.5 & 383.6 & 439.2 \\
\hline No cubiertos / Costos y gastos & $46 \%$ & $46 \%$ & $9 \%$ & $17 \%$ \\
\hline
\end{tabular}

Comparando las tarifas equivalentes por $20 \mathrm{~m}^{3}$ para estas seis organizaciones se observa en la Figura 5 que es la empresa grande (EMCALI) la que genera las mayores tarifas, lo que es similar en otras zonas del país (CadavidGiraldo 2008).

Esto llama mucho la atención especialmente en el contexto de los argumentos esgrimidos por los organismos multilaterales que ponen la atomización del sector y las bajas economías de escala como explicación al mal desempeño del sector de abastecimiento rural de agua (Krause 2009). Es claro que para cada situación particular de acceso al agua, topografía, capacidad de pago de la población atendida, capacidad técnica disponible entre otros factores, existe un tamaño o nivel de agrupación que permite mayores eficiencias y que existe un punto de inflexión por debajo del cual las organizaciones prestadoras son inviables. Pero como hemos demostrado con estos ejemplos, organizaciones pequeñas como La Sirena con 900 conexiones en una zona de alta complejidad topográfica, no solo son viables financieramente sino que además mantienen tarifas por debajo de las ofrecidas en las zonas urbanas. Y organizaciones como Acuabuitrera y Acuasur (entre 1600 y 2500 suscriptores) con altos niveles técnicos, también mantienen costos muy por debajo de lo que se esperaría para sus zonas de alta complejidad biofísica. Esto demuestra que las organizaciones comunitarias pueden ser una solución financieramente más eficiente que las grandes empresas público/privadas y sus economías de escala para suplir agua potable en zona rural a bajo costo. 
Es posible que una vez las organizaciones comunitarias empiecen a recibir los subsidios sus costos tengan incrementos que reflejen la inclusión de los costos que hasta el momento son subsidiados por la comunidad como se mostró en la Tabla 6, o que haya una mayor inclusión de costos de inversión. Aun así, el caso de Tribunas Córcega que ya recibe los subsidios muestra que las organizaciones comunitarias no tienen costos más altos, lo que es un indicador de que la rentabilidad del capital es un factor importante en el mayor costo del servicio de acueducto en las zonas urbanas.

Tabla 6. Comparación de cargos fijos y por consumo para 5 organizaciones comunitarias y EMCALI (CMA en pesos por mes, $\mathrm{CC}$ en pesos por $\mathrm{m}^{3}$ ).

\begin{tabular}{lrr}
\hline Organización & CMA & CC \\
\hline Tribunas & 15,000 & 617 \\
Acuabuitrera & 7,556 & 725 \\
Golondrinas & 4,919 & 735 \\
La Sirena & 4,008 & 388 \\
Acuasur & 3,998 & 741 \\
Emcali & 8,060 & 1,324 \\
\hline
\end{tabular}

Adicionalmente parece injusto que siendo la rentabilidad al capital un factor de incremento en las tarifas urbanas, les sea permitido a las organizaciones comerciales incluirla como parte de los subsidios que terminan siendo cubiertos por el Estado, cuando una porción tan pequeña de las comunidades rurales están recibiendo los subsidios para cubrir los costos de operación y administración de sus sistemas.

\section{El riesgo de la dependencia y de la competitividad}

Algunas organizaciones comunitarias prefieren mantener sus ingresos exclusivamente de las tarifas como el caso de La Sirena. Argumentan que la dependencia de los subsidios implica una alta vulnerabilidad a un flujo de recursos incierto. Si se tienen en cuenta los retrasos de los municipios en el pago efectivo de estos subsidios y los análisis sobre el modesto aunque no insignificante efecto que tienen los subsidios en los gastos de los más pobres (Kumives et al. 2005), no les falta razón. Algunos administradores de sistemas comunitarios consideran que el influjo de fondos provenientes del Estado hace que se generen expectativas económicas en las comunidades y que se pierda el espíritu comunitario y de solidaridad que caracteriza estos sistemas. Para otras organizaciones, el acceso a los subsidios puede convertirse en la salvación de sistemas comunitarios cuyos administradores voluntarios están entrando en edades de retiro en zonas donde las nuevas generaciones aspiran a obtener empleo remunerado $\mathrm{y}$ acceder a espacios de capacitación formal como el caso de Golondrinas.

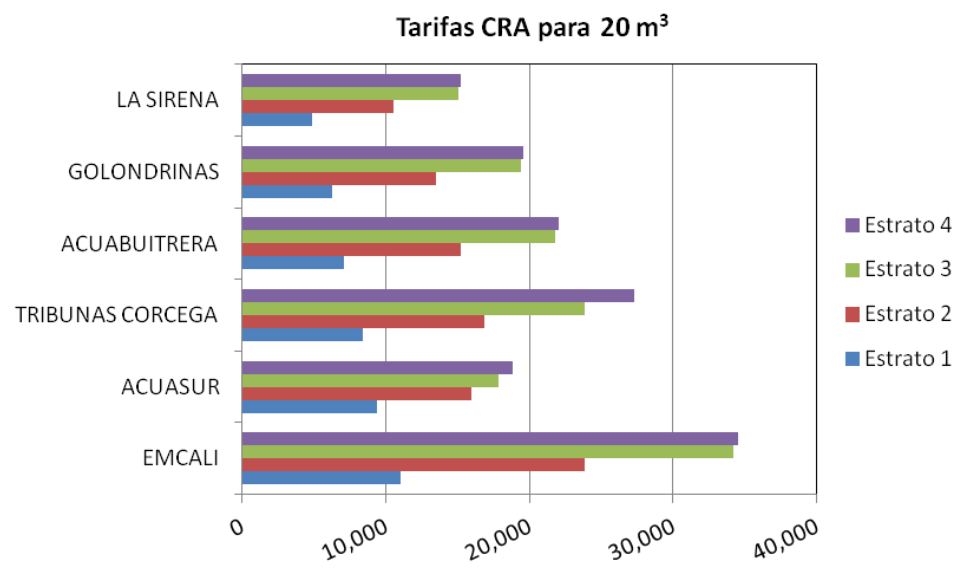

Figura 5. Tarifas CRA por estrato para $20 \mathrm{~m}^{3}$ mensuales (cinco organizaciones comunitarias y EMCALI).

Entre estas dos posturas se debaten muchas organizaciones que ven razones válidas en ambos argumentos. Pero mientras tanto los subsidios que provienen del Estado siguen siendo asignados desproporcionadamente a favor de organizaciones comerciales, pues las comunitarias no han alcanzado un nivel de gestión que les permita acceder a los medios para cumplir con los requisitos legales, o cuando alcanzan ese nivel, se encuentran con obstáculos burocráticos como la estratificación o las demoras en los procesos. Este es el caso de Acuasur que presentó al FSRI toda la documentación exigida por la ley durante cuatro años sin obtener respuesta por parte del municipio, hasta que finalmente recibió una respuesta positiva en 2014.

Por otro lado, algunos sistemas que reciben los subsidios como el caso de Tribunas Córcega perciben otro tipo de riesgo. Al convertirse en organizaciones viables y que producen excedentes para invertir en las comunidades, han pasado a ser organizaciones atractivas para los municipios que como Pereira ha manifestado su interés en integrar este sistema a la empresa de servicios públicos de la ciudad. En este riesgo se evidencia la tensión entre dos modelos viables 
de gestión del agua, basados en culturas, ecologías y economías diferentes (Escobar 2006).

\section{CONCLUSIONES}

Mucho se ha dicho sobre la brecha que separa la regulación para las organizaciones comunitarias del agua y sus capacidades para cumplir con estas normas. Tanto la Defensoría del Pueblo (DDP 2011) como organismos internacionales como la CEPAL (Carrasco-Mantilla 2011) han llamado la atención a las entidades que hacen políticas sobre agua y saneamiento para que ajusten las reglas de juego a las condiciones de las organizaciones rurales para que puedan tener acceso a los mismos derechos a los que acceden las organizaciones empresariales. El esquema de subsidios al agua en las zonas rurales no se maneja como un subsidio a los más pobres, sino como una recompensa a las comunidades que logran organizar un sistema de abastecimiento con un sistema contable y de información suficiente como para cumplir con los requisitos exigidos por la ley.

El uso del método CRA es un indicador de la sostenibilidad financiera de la operación y administración de los sistemas a través de las tarifas, pero al igual que otros requisitos para el acceso al FSRI como por ejemplo el reporte de información al SUI, requieren de conocimientos contables y de acceso a computadores e internet, que en gran parte de las zonas rurales no son comunes. Por otro lado, para las organizaciones comerciales la ley permite el cobro de los márgenes de rentabilidad financiera sobre las inversiones causadas (públicas o privadas) lo que ha llevado al incremento en las tarifas por parte de las empresas grandes que prestan el servicio en zonas urbanas.

Este contraste entre el tratamiento dado a las organizaciones comunitarias sin ánimo de lucro y a las empresas comerciales no solo ha hecho que los principios de solidaridad y redistribución de ingresos no se hayan llevado a la práctica, sino que por el contrario han generado una mayor brecha entre zonas urbanas y rurales. En las zonas urbanas los pobres están pagando tarifas más altas que en las zonas rurales, a pesar de que las empresas urbanas reciben los subsidios del Estado. Los sistemas comunitarios se debaten entre conservar su independencia y autosuficiencia sin contar con recursos del Estado, a los que sin embargo sus comunidades tienen derecho, y la lucha por obtenerlos para poder garantizar su sostenibilidad en el largo plazo.

Como resultado estas organizaciones dependen del trabajo voluntario de miembros de la comunidad y no pueden costear el mantenimiento y reposición de infraestructura, manteniendo una alta vulnerabilidad técnica y financiera. El acceso equitativo al FSRI por parte de todas las organizaciones que prestan los servicios de agua es necesario para avanzar en el cumplimiento del derecho humano al agua, para hacer sostenibles los sistemas comunitarios de servicios de agua y saneamiento y para reducir la enorme brecha entre zonas urbanas y rurales. La ley colombiana ofrece oportunidades para que las organizaciones comunitarias luchen por el acceso a los subsidios, pero la gran mayoría están muy lejos de alcanzar el nivel de gestión que se requiere para poder emprender esta lucha.

\section{REFERENCIAS}

ALZATE, M.C. 2006. La estratificación socioeconómica para el cobro de los servicios públicos domiciliarios en Colombia ¿Solidaridad o focalización? CEPAL. Naciones Unidas, Santiago de Chile. 101 pp.

CADAVID-GIRALDO, N. 2008. Agua para consumo doméstico en Colombia: costos y regulación tarifaria. Gestión y Ambiente, 11(1): 97-108.

CARRASCO-MANTILLA, W. 2011. Políticas públicas para la prestación de los servicios de agua potable y saneamiento en las áreas rurales. Comisión Económica para América Latina y el Caribe (CEPAL), Colección documentos de proyectos LC/W. Santiago de Chile, 57 pp.

DEFENSORÍA DEL PUEBLO (DDP). 2011. Acueductos comunitarios en la zona rural de Ciudad Bolívar. Defensoría del Pueblo de Colombia. Imprenta Nacional, Bogotá. 126 pp.

ESCOBAR, A. 2006. Difference and conflict in thestruggleover natural resources: a political ecology framework. Development, 49(3): 6-13.

FERNÁNDEZ, D. 2004. Sector agua potable, Informes de base. Colombia: Desarrollo económico reciente en 
infraestructura (REDI). Banco Mundial,Washington, D.C. $118 \mathrm{pp}$.

KRAUSE, M. 2009. The political economy of water and sanitation. Routledge, New York. 252 pp.

KUMIVES, K. FOSTER, V. HALPERN, J. \& WODON, Q., 2005. Agua, electricidad y pobreza: ¿quién se beneficia de los subsidios a los servicios públicos? Banco Mundial, Mayol Ediciones S.A., Bogotá. 318 pp.

MARTÍNEZ-ALONSO, L.C. 2004. Revisión del modelo de estratificación socioeconómica aplicado en Bogotá, D.C. Tesis de Maestría, Universidad Nacional de Colombia.

MELÉNDEZ, M. 2008. Subsidios al consumo de los servicios públicos: reflexiones a partir del caso Colombiano. Perspectivas 6(1): 3-40.

MINA-ROSERO, L. 2004. Estratificación socioeconómica como instrumento de focalización. Economía y Desarrollo 3(1): 54-67.

PÉREZ M.A. 2001. Balance y gestión en empresas de servicios de acueducto y alcantarillado de pequeña escala en Colombia. Análisis comparado para diferentes formas organizativas y escalas de servicio. CINARA, Cali, 29 pp.

TAMAYO, S.P. \& GARCÍA, M. 2006. Estrategia estatal para el fortalecimiento de entes prestadores de servicios públicos en el pequeño municipio y la zona rural. El programa cultura empresarial adelantado en Colombia. En Quiroz, F., Faysse, N. y Ampuero, R. Apoyo a la gestión de Comités de Agua Potable; experiencias de fortalecimiento a comités de agua potable con gestión comunitaria en Bolivia y Colombia. Cochabamba, Bolivia: Centro Agua/UMSS. 